\title{
Sensor-Selbsttest und -Diagnose
}

\author{
K Peter Seefeld, \\ Endress+Hauser Wetzer GmbH + Co. KG, D-87484 Nesselwang, Obere Wank 1
}

\section{Zusammenfassung}

Sensor-Selbsttest-Methoden und Diagnoseverfahren finden in der Prozessmesstechnik wie in der Prozessanalysenmesstechnik (PAT) seit über einem Jahrzehnt eine verstärkte Beachtung.

Ergebnisse eines Sensor-Selbsttests bzw. eines Sensor-Diagnoseverfahrens müssen weitestgehend verlässlich sein, um eine korrekte Funktionsfähigkeit über die Sensorlebensdauer signalisieren zu können.

Unter Kosten/Nutzen-Überlegungen sollten Messwertaufnehmer-Systeme mit Selbsttest-Einrichtungen höhere Verfügbarkeiten und geringere Ausfallraten gewährleisten als reine Messwertaufnehmer ohne derartige Einrichtungen.

Für Selbstüberwachung und Diagnose werden sensorielle Hilfsgrößen genutzt sowie zusätzliche physikalische Sensorbewertungen eingesetzt, die eine redundante Funktionalität zum Erzielen einer höheren Fehlertoleranz aufweisen.

Ein auf sensorieller Ebene diversitär redundante Verfahren zur Bestimmung der Temperatur mittels Rauschthermometrie wird detailliert vorgestellt und daneben eine diversitär redundante Methode mittels miniaturisierter Fixpunktzellen gezeigt. Fachkreise in Normungs- und Verbandsgremien befassen sich mit Begriffsdefinitionen und Bewertungskriterien für Selbstüberwachung und Diagnose, wie in einer kurzen Übersicht vorgestellt wird.

\section{Autarke Selbstüberwachungsfunktion}

Im Rahmen dieses Beitrags sollen zunächst autarke Sensorfunktionen im Mittelpunkt stehen, wobei "in situ"Verfahren dadurch gekennzeichnet sind, dass das Sensorelement, das eine Sensor-Selbsttest-Funktionalität aufweisen soll, sich unmittelbar im Prozessmedium befindet. Aus der Sicht einer "Leitstelle" sind derartige Prüfverfahren auf der "unteren" Feldgeräteebene direkt in der Prozessebene angesiedelt.

Nach Möglichkeit soll ein derartiges Prozess-nahes System selbst in Grenzbetriebssituationen noch hinreichend geringe Messwertunsicherheiten aufweisen.

Auftretende „Fehler“ eines Sensorelementes, die zur Überschreitung einer spezifizierten Messunsicherheit führen, sind als Störungen aufzufassen, demnach weist das beobachtete Sensorelement in der Folge Messabweichungen auf, die außerhalb eines zulässigen Toleranzbandes liegen [2]. Derartige Zustände, die als andauernde oder zeitweise Störungsfälle irreversibel oder reversibel auftreten, sind mittels einer im autarken Sensor vorhandenen redundanten Sensor-Funktionalität zu erfassen [1].

Durch Sensor-Redundanz wird eine kleinere Messunsicherheit, z. B. durch Mittelwertbildung ermöglicht [3]. Wird dafür ein zusätzliches Sensorelement eingesetzt, das mit dem ersten Sensorelement identisch ist, so liegt eine homogene Redundanz vor, mit der indessen bestimmte systematische Störungsfälle nicht zu erfassen sind. Zusätzlich steigt bei identisch redundant eingesetzten Sensorelementen das gesamte resultierende Ausfallrisiko, wie anhand einer multiplikativen Verknüpfung der identischen Einzelzuverlässigkeiten zur Gesamtzuverlässigkeit RG(t), erkannt wird. Die Zuverlässigkeit des ,primären“ Sensorelementes, wird mit RS(t) bezeichnet, die Zuverlässigkeit des überwachenden redundanten Elementes mit $\mathrm{RÜ}(\mathrm{t}) \cdot \mathrm{RG}(\mathrm{t})=\mathrm{RS}(\mathrm{t}) \cdot \mathrm{R} \ddot{U}(\mathrm{t})$ mit $\mathrm{RS}(\mathrm{t}), \mathrm{R} \ddot{U}(\mathrm{t})<1$ und damit $\mathrm{RG}(\mathrm{t})<\mathrm{RS}(\mathrm{t}), \mathrm{R} \ddot{U}(\mathrm{t})$

In weiterführender, statistischer Betrachtung weist ein zwei aus drei - Auswahlsystem aus homogen redundanten Sensorelementen eine geringere Messunsicherheit auf als ein Einzelsensor, falls insbesondere

Es sei denn für die Auswahl existiert eine Auswahlfunktion = Voter-Funktion vorhanden ist, die nicht systematischen Störungen unterliegt und unabhängig von den drei zur Auswahl stehenden Sensorelementen einen abgesicherten Voter-Wert aufweist, der sich dem Wert eins nähert.

Dies könnte beispielsweise von einer diversitär redundanten Sensorfunktion geleistet werden.

Das zwei aus drei Auswahlverfahren würde dabei die zusätzliche Hinzuziehung eines vierten diversitären Sensorelementes fordern, was einen relativ hohen Gesamtaufwand darstellt. Prinzipiell wird dabei unter Einbeziehung der korrekt erkannten Sensorstörungen die Gesamtzuverlässigkeit erhöht, nicht indessen die zu 
erwartende Verfügbarkeit der Messung entscheidend gesteigert, da die drei baugleichen Sensoren, deren Messsignale zur Auswahl stehen, zeitlich und applikationsbezogen ähnliche Ausfalleigenschaften aufweisen werden.

Hier sind nicht zuletzt unter wirtschaftlichen Gesichtspunkten geeignetere Lösungen anzustreben.

Neben einer diversitären Funktionalität zur Störfallanalyse sollte eine zusätzliche robuste Sensorfunktion idealerweise gleichzeitig eine metrologisch verwertbare Messgröße bereitstellen. Beim erkannten und andauerndem Ausfall der primären Sensor-Messgröße kann diese ausgegeben werden, wobei gleichzeitig analysiert wird, ob die primäre Sensorfunktion noch grundsätzlich vorhanden ist oder beispielsweise mit diversen Schäden behaftet vor dem zeitabhängigen Totalausfall steht.

\subsection{Rauschthermometrie}

Das thermische Rauschen in elektronischen Leitern wird als primäre metrologische Methode für prozessmesstechnische Spezialanwendungen bereits seit ungefähr drei Jahrzehnten eingesetzt.

In Rauschthermometrie-Einrichtungen für Hochtemperaturmessungen sind beispielsweise Widerstandsthermometer aus Wolfram-Rhenium Legierungen und aus inertisierten Graphit-Sensoren realisiert worden.

Die Messung der thermischen Rauschspannung, die an einem Rtd-Messwiderstand abgegriffen wird, bietet eine redundante Sensormessgröße neben einer primären Widerstands- (Rtd) oder Thermoelement (TC)Messung.

Im Gegensatz zum Driftverhalten eines ohmschen Widerstandswertes bleibt die Rauschleistung am Messwiderstand unbeeinflusst von strukturellen Änderungen des Widerstandselementes, die gegebenenfalls unter drastischen Einsatzbedingungen auftreten können, so dass eine diversitäre Redundanz hier ebenso gegeben ist.

\subsubsection{Rauschthermometrie Methodik Messverfahren}

Der quadratische Mittelwert einer thermischen Rauschspannung über einem Widerstand mit der Impedanz $\mathrm{Z}$ bei einer Temperatur $\mathrm{T}$ ist durch die Nyquistformel darstellbar. Bei Frequenzen kleiner $1 \mathrm{MHz}$ und Temperaturen größer $25 \mathrm{~K}$ gilt die nebenstehende angenäherte Beziehung bei einer Abweichung kleiner 1ppm.

$$
d \overline{u_{T}^{2}}=4 h f \operatorname{Re}(Z)\left[\frac{1}{2}+\frac{1}{e^{\frac{h f}{k T}}-1}\right] d f \quad \overline{u_{T}^{2}}=4 k T R \Delta f
$$

Für einen Widerstand $\mathrm{R}($ sample $)=100 \Omega$, bei Raumtemperatur $\mathrm{T}=300 \mathrm{~K}$ und einer Messbandbreite von $\mathrm{f}=100 \mathrm{kHz}$ ergeben sich für die Rauschleistung $1, \mathbf{1} \mathbf{1 0}^{-13} \mathbf{V}^{2}$, entsprechend einer mittleren Rauschspannung von $\mathbf{4} \mathbf{1 0}^{-7} \mathrm{~V}$.

Zur Erfassung der geringen Rauschspannung erweist sich unter prozessmesstechnischen Randbedingungen ein Korrelationsverfahren in Verknüpfung mit einer Referenzmethode als vorteilhaft, bei der wechselseitig der Mess- und der Referenzwiderstand mit den Vorverstärkereingängen beschaltet werden.

Die frequenzabhängige Verstärkung A(f), die u.a. von der Umgebungstemperatur beeinflusst wird, geht bei diesem Verfahren nicht absolut als Messunsicherheit in den Messwert ein.

Mit dieser Methode sind unkorrelierte, parasitäre Rauschsignale, wie Kabeleinstreuungen oder das Eigenrauschen der Operationsverstärker weitgehend zu unterdrücken [7].

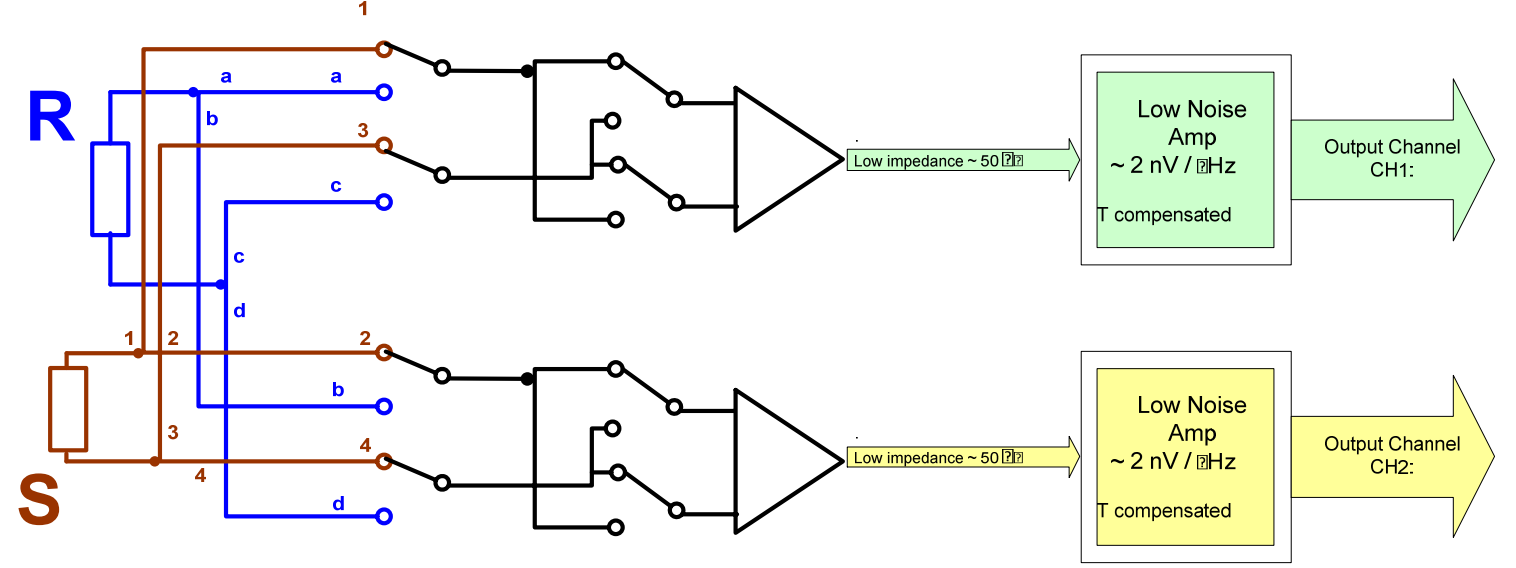

Bild 1 wechselseitige Umschaltung von Mess (S)- und Referenzwiderwtand (R) an die Verstärkerkanäle 


\subsubsection{Rauschthermometrie Signalverarbeitung}

Für die Leistungsdichtespektren der Rauschsignale über der Frequenz f gilt:

$$
\begin{aligned}
& W_{u u, a}(f)=G_{a}(f)^{2} 4 k T_{s} R_{s}+N_{a}(f) \\
& W_{u u, b}(f)=G_{b}(f)^{2} 4 k T_{s} R_{s}+N_{b}(f)
\end{aligned}
$$

Dabei werden mit $\mathrm{Ga}(\mathrm{f})$ und $\mathrm{Gb}(\mathrm{f})$ die frequenzabhängigen Verstärkungen bezeichnet und $\mathrm{Na}(\mathrm{f})$ und $\mathrm{Nb}(\mathrm{f})$ stehen für die Leistungsdichten der parasitären Rauschspannungsquellen im betrachteten Frequenzbereich. $\mathrm{Na}(\mathrm{f})$ und $\mathrm{Nb}$ (f) fallen nach einer Kreuzkorrelation im Frequenzbereich heraus, da die parasitären Rauschsignale der beiden Kanäle zueinander unkorreliert sind.

Das Sensorrauschsignal ist dagegen, sofern zeitgleich abgetastet wird, weitgehend korreliert und damit ergibt sich durch Kreuzkorrelation das Leistungsdichtespektrum des Sensorrauschsignals zu:

$$
W_{u x s}(f)=F_{a}^{*}(f) F_{b}(f)=G_{a b}(f)^{2} 4 k T_{s} R_{s}
$$

$\mathrm{a}(\mathrm{f})$ bezeichnet die konjugierte Fouriertransformierte des Rauschsignals aus Kanal A und mit b(f) wird die Fouriertransformierte des Rauschsignals aus Kanal B bezeichnet.

\section{SIGNAL PROCESSING}
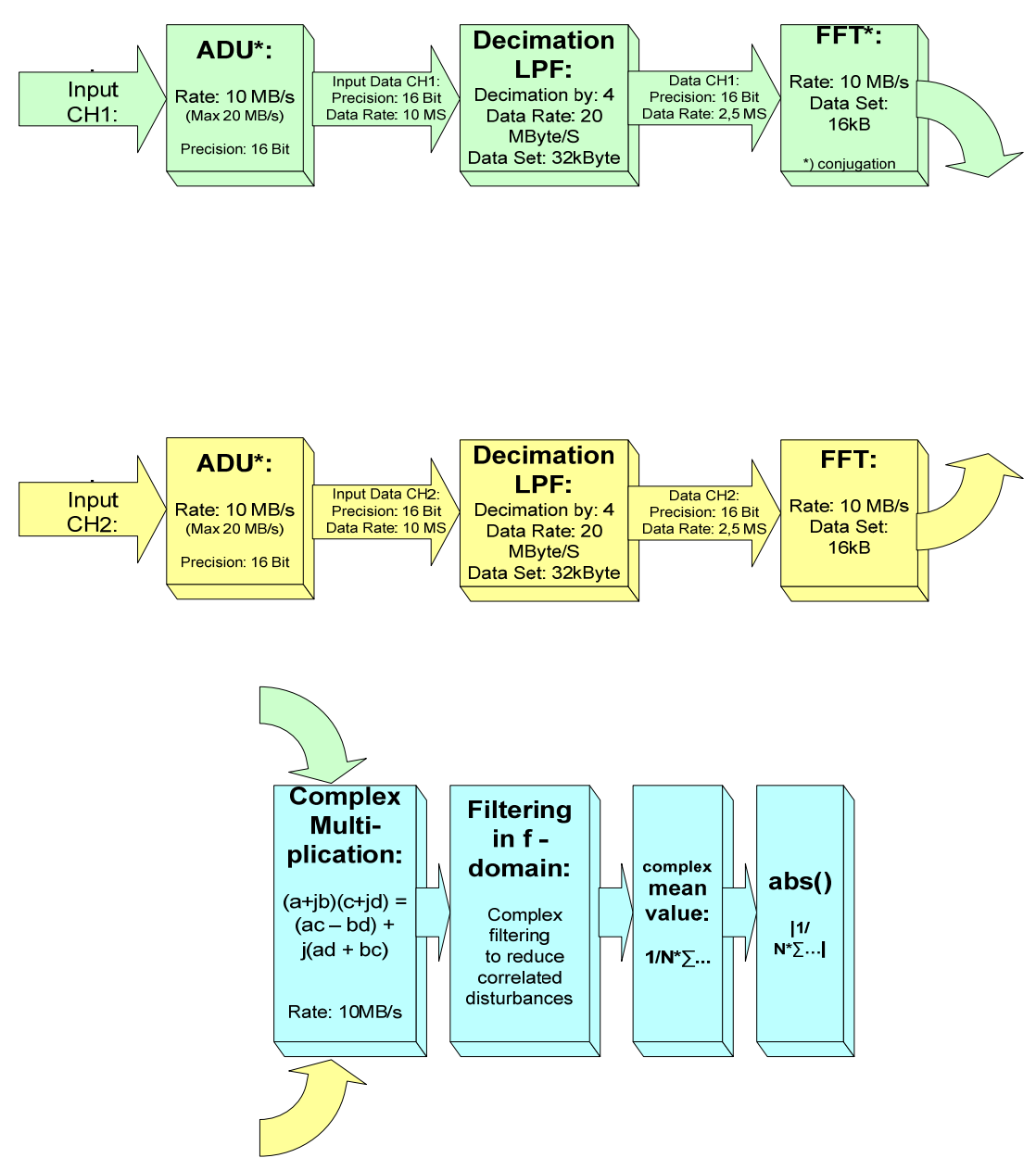

Bild 2 Blockschaltbild für das angewendete Korrelations- und Vergleichsverfahren

\subsubsection{Messung der Leistungsdichtespektren}

In Bild 5 wird ein gemitteltes Leistungsdichtespektrum gezeigt, das in einem Laboraufbau nach 839 Millisekunden Messzeit aus 512 koaddierten Spektren im Frequenzbereich bis $625 \mathrm{KHz}$ entstand [6]. Dabei beträgt die Korrelationsbandbereite $625 \mathrm{kHz}-100 \mathrm{kHz}=525 \mathrm{kHz}$ und die Messzeit $\mathrm{t}_{\mathrm{m}}=0,839 \mathrm{~s}$. woraus eine statistische Messunsicherheit von 0,006 entsprechend 0,6\% resultiert. 


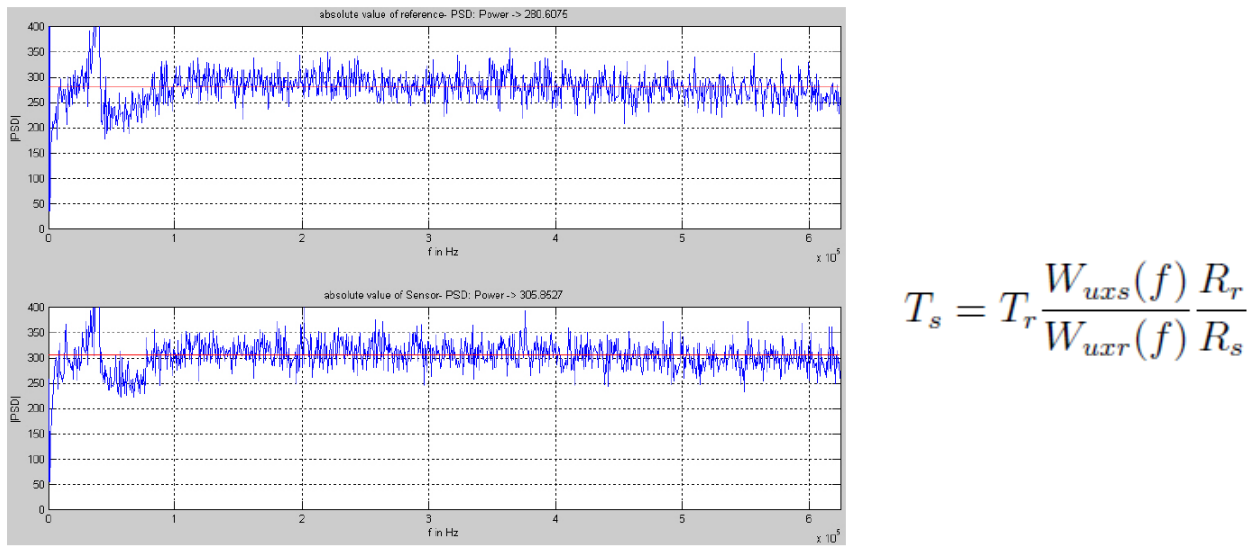

Bild 3 In 0,839 Sekunden Messzeit aus 512 Spektren gemitteltes Leistungsdichtespektrum Oben für Referenz; $0^{\circ} \mathrm{C}$, Eiswasser / Unten für die unbekannte Messwiderstandstemperatur Aus dem Verhältnis der Leistungsdichtespektren und der bekannten Referenztemperatur wird unmittelbar die Sensortemperatur von $24,57^{\circ} \mathrm{C}$ erhalten bei einer Abweichung von $-0,63{ }^{\circ} \mathrm{C}$, gegenüber einer direkten Widerstands (Rtd)-Vergleichsmessung, deren Messwert 25,2 ${ }^{\circ} \mathrm{C}+/-0,05$ beträgt.
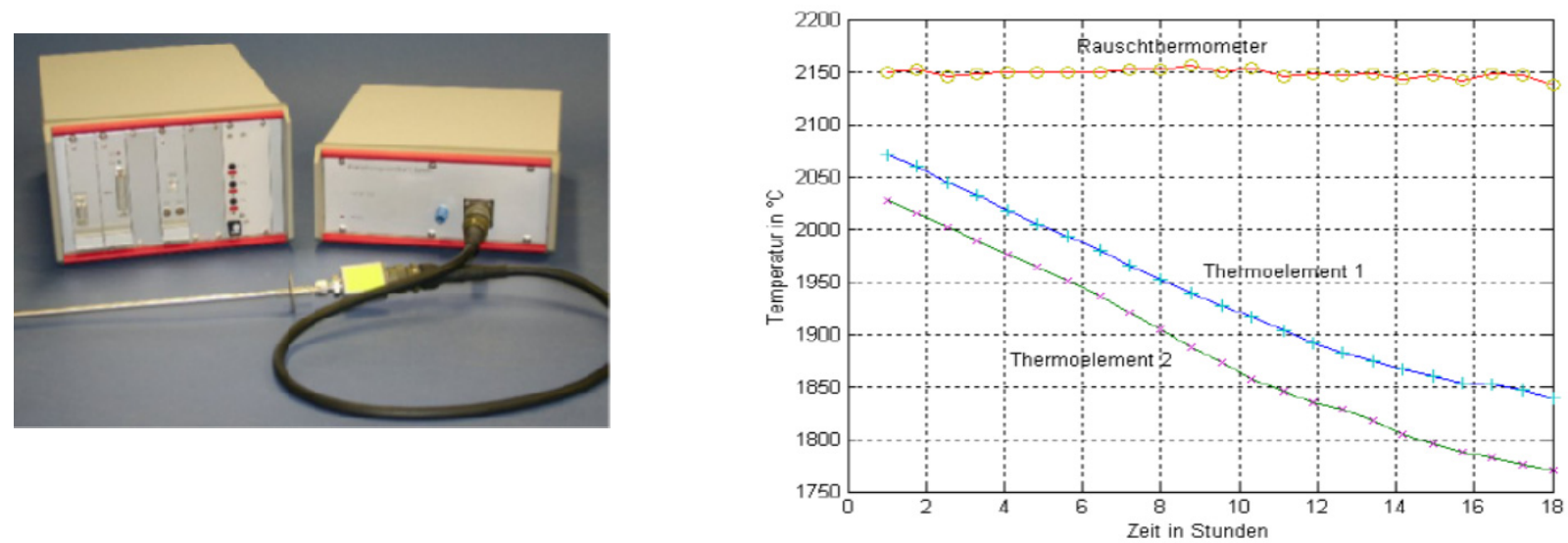

Bild 4 Für technische Spezial-Anwendungen als robustes Messverfahren in 2006 als Prototyp eingeführt: Rauschthermometer, Typ 6 nach dem Umschaltungs-Korrelationsverfahren, Zimmermann E., Glaas W. et. al. Forschungszentrum Jülich mit nebenstehender Vergleichsmessreihe, TC-Elemente in metallurgischer Hochdruckanwendung.

\subsection{Miniaturisierte Fixpunktzellen}

Phasenumwandlungstemperaturen von Reinsubstanzen, wie sie in metrologischen Kalibrierlabors als ITS90 Fixpunkte bekannt sind, werden zu Rückführungszwecken herangezogen.

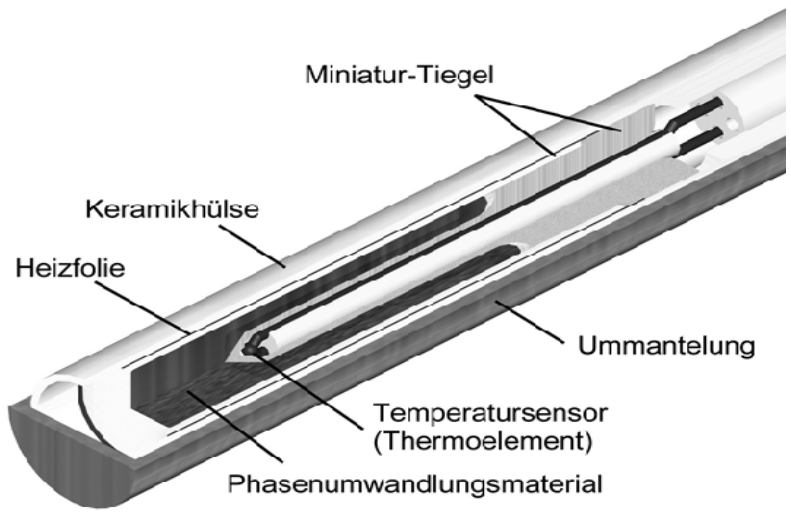

Bild 5 Schnittzeichnung einer integrierten miniaturisierten Fixpunktzelle 
Für Prozessanwendungen werden miniaturisierte Keramikkompartimente beispielsweise in

Kontaktthermometern eingebaut, die als sogenannte Miniaturfixpunktzelle kleinere Mengen einer

Reinsubstanz enthalten [4]. Derartige in Prozess-Thermometern eingesetzte miniaturisierte Fixpunktzellen

erfahren im Allgemeinen eine relativ rasche Phasenumwandlung, die entsprechenden Temperaturänderungen

folgt.

Gebräuchliche Fixpunktzellen im metrologischen Kalibrierlabor weisen dagegen eine langzeitig über mehrere Stunden andauernde Phasenumwandlung auf, die eine annähernde Einstellung eines thermischen Gleichgewichtes zulässt, woraus sich unmittelbar die Fixpunkttemperatur zurückführen lässt.

Die relativ kurze Dauer der Phasenumwandlung in miniaturisierten Fixpunktzellen unterliegt demgegenüber vielfältigen Einflüssen. Dazu gehören, bauartbedingte Wärmeableitungseffekte an den

Keramikkomponenten, Wärmeleitungseffekte am Thermometereinsatz bzw. am Schutzrohr, das üblicherweise thermisch mit dem Messmedium gekoppelt ist.

Gegebenenfalls wird eine zusätzliche Substratheizung eingesetzt, um bei einer Thermometertemperatur knapp unterhalb der Fixpunkttemperatur die Schmelzphase in einer Miniaturfixpunktzelle einzuleiten, wobei es zahlreicher Optimierungen bedarf, um eine zumindest hinreichende thermische Gleichgewichtseinstellung zu erzielen.

Zur Bewertung derartiger komplexer thermischer Kopplungen dienen teilweise aufwändige numerische Näherungsverfahren.

Als einfache Näherungsmethode dient eine Geradenapproximation, die zur Bestimmung der

Fixpunkttemperatur herangezogen werden kann [5].

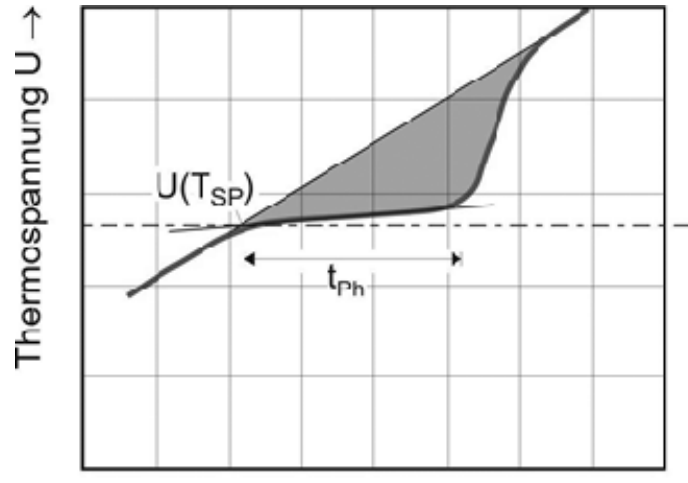

Zeit $\mathrm{t} \rightarrow$

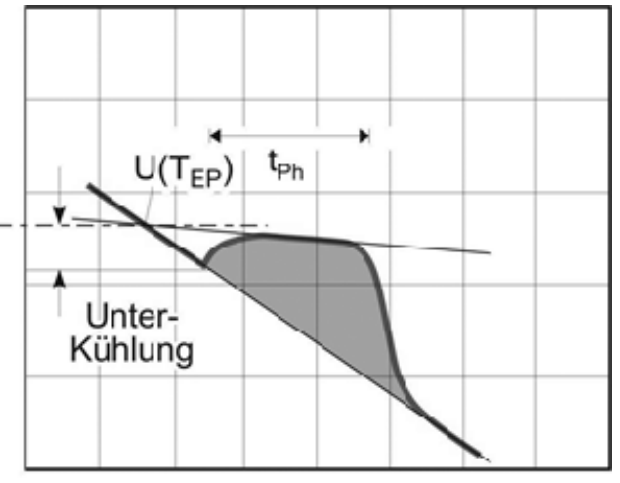

Zeit $\mathrm{t} \rightarrow$

Bild 6 Abschnittsweise werden die gemessenen Temperaturverläufe mittels Geraden approximiert und an den jeweiligen Schnittpunkten die Anfangstemperatur für den Schmelzpunkt bzw. die Endtemperatur für den Erstarrungspunkt bestimmt

\section{$2 \quad$ Standardisierung}

In einigen Fachkreisen und Standardisierungsgremien werden Kriterien und Bewertungsansätze für Selbstüberwachungs- und Diagnose-Verfahren erarbeitet.

Die folgende Namur-Empfehlung wurde als Richtlinie verabschiedet, während auf normativer Ebene die britische Norm BS7986 keine internationale Zustimmung als IEC-Norm fand.

\subsection{NAMUR Empfehlung 107, VDI/WIB-Richtlinie 2650}

Die NAMUR (Arbeitsfeld 3, AK3.1) und der VDI (Verein Deutscher Ingenieure) im Verbund mit der WIB; (International Instrument Users' Association WIB, NL) haben gemeinsam abgestimmte Empfehlungen veröffentlicht, die als Namur-Empfehlung NE 107 und als VDI/WIB-Richtlinie 2650 bekannt wurde. Nach diesem empfohlenen Schriftstück sollen alle von einem Feldgerät erhaltene Diagnosedaten als Ergebnisse der Selbstüberwachung auf eines der vier Statussignale abgebildet werden:

\subsubsection{Ausfall (F)}

Aufgrund einer Funktionsstörung im Feldgerät oder an seiner Peripherie ist das 
Ausgangssignal ungültig. Bezeichnet den Zustand einer Einheit, in dem sie unfähig ist, eine geforderte Funktion zu erfüllen, ausgenommen die Unfähigkeit während der Wartung oder anderer geplanter Maßnahmen oder infolge des Fehlens äußerer Mittel , z. b. "kein Messsignal" vorhanden.

\subsubsection{Wartungsbedarf (M):}

Das Ausgangssignal ist zwar noch gültig, aber der Abnutzungsvorrat wird demnächst erschöpft oder aufgrund von Einsatzbedingungen eine Funktion in Kürze eingeschränkt sein, z. B. Alterung der pHElektrode

\subsubsection{Außerhalb der Spezifikation (S)}

Vom Gerät durch Selbstüberwachung ermittelte Abweichungen von den zulässigen Umgebungs- oder Prozessbedingungen oder Störungen im Gerät selbst weisen darauf hin, dass die Messunsicherheit bei Sensoren oder Sollwertabweichung bei Aktoren wahrscheinlich größer ist als unter Betriebsbedingungen zu erwarten.

\subsubsection{Funktionskontrolle (C)}

Am Feldgerät wird gearbeitet, das Ausgangssignal ist daher vorübergehend ungültig (z.B. eingefroren)

Zusätzlich werden Prioritäten festgelegt. Der Status “Ausfall (F)" hat eine höhere Priorität als der Status "Wartungsbedarf (M)".

Der Status "Wartungsbedarf (M)" weist eine höhere Priorität auf als der Status "Außerhalb der Spezifikation (S).

Diagnose-Angaben die dem Status " Funktionskontrolle (C)" zugeordnet werden besitzen die niedrigste Priorität.

\subsection{British Standard BS7986}

Die britische Norm BS7986 beinhaltet eine Metrik zur Erfassung der Datenqualität von Messdaten zu Status des Messwertes und zu der damit verknüpften Messunsicherheit. Während die NE 107 auf Feldgeräte in einer Gesamtbetrachtung aus Anwenderperspektive ausgerichtet ist wird die in der spezifizierten BS7986 Qualitäts-Metrik auf jeden einzelnen Messkanal des Feldgerätes bezogen. Bei dieser Qualitäts-Metrik nach BS7986 werden Unterscheidungen getroffen zwischen analogen Messungen (AI), diskreten Parametern (DI), analoger Regelung und Parameter für Aktoren (AO), diskrete Regelung und deren Parameter (DO). In der BS7986 wird jeder erfasste Wert mit einem Parameter zur Angabe der Unsicherheit verknüpft. Für aufkommenden Wartungsbedarf werden zusätzliche Parameter festgelegt.

\section{Tafel 1 Ausgabefunktionen eines SEVA - Sensors}

VMV

VU

MV Status

Device Status
Validierte Mess-Werte

Die validierte Unsicherheit des VMV

Wie der aktuelle VMV ermittelt wurde

Den Zustand des physikalischen Gerätes

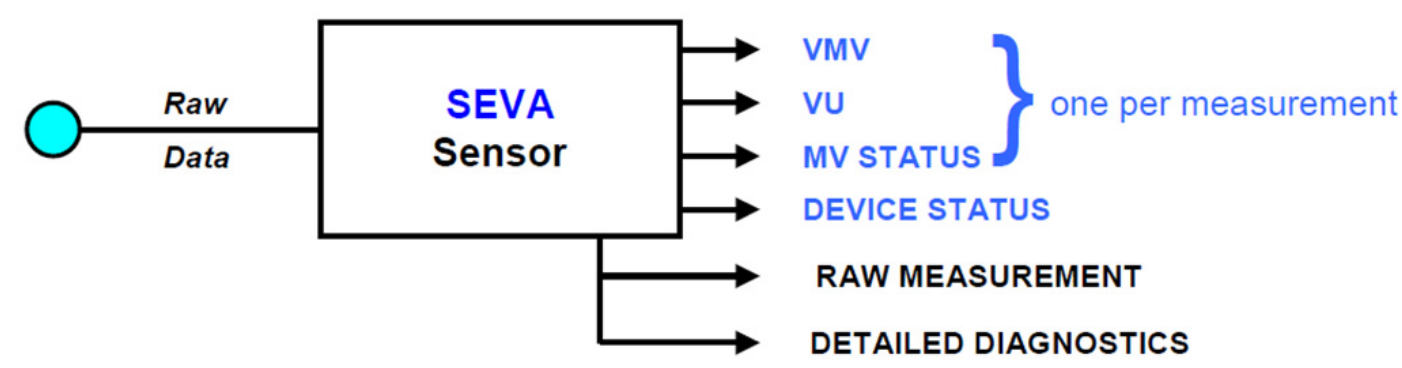

Bild 8 Informationen, die ein SEVA Sensor bereitstellen soll; (siehe oben: englischer Originaltext) 


\section{Literatur}

[1] R. Werthschützky, R. Müller, M. Nuber: Selbstüberwachung und Störungstoleranz von Sensoren, Sensoren und Messsysteme 2006, Berlin Offenbach, VDE Verlag

[2] F. Mesch: Strukturen zur Selbstüberwachung von Messsystemen, atp Automatisierungstechnische Praxis 43. Jahrgang, 8/2001, S. 62-67, München, Oldenbourg Verlag

[3] R. Werthschützky, R. Müller: Selbstüberwachung und Störungstoleranz von autarken Sensoren, tm Technisches Messen 74 4/2007 S.176-184, München, Oldenbourg Verlag

[4] F. Bernhard, D. Boguhn, S. Augustin, H. Mammen, A.Donin: Application of self-calibrating thermocouples with miniature fixed-point cells in a temperature range from $500{ }^{\circ} \mathrm{C}$ to $650{ }^{\circ} \mathrm{C}$ in steam generators, in: Ilic, D.: IMEKO 2003, Dubrovnik: International Measurement Confederation Hrvatsko, 2003 [5] F. Bernhard, S. Augustin, H. Mammen: Application of self-calibrating thermometers with miniature fixed-point cells in a temperature range from $300^{\circ} \mathrm{C}$ to $650^{\circ} \mathrm{C}$, in: D.ZVIZDIC, D.: TEMPMEKO 2004, Zagreb : University of Zagreb, FSB, 2004, S. 1285

[6] P. Seefeld, S. Damith, M. Konrad, M. Korn, A. Zotz: Prozessgeeignete

Rauschtemperaturmessungen, Sensoren und Messsysteme 2010, Berlin Offenbach, VDE Verlag

[7] H. Brixy, J. Hecker, J. Oehmen, K. F. Rittinghause, H.Howener, W. Setiawan, E. Zimmermann: Noise Thermometry for industrial and metrological applications at KFA Jülich, in: Temperature, Its Measurement and Control in Science and Industry, 6, 993-996, J. F. Schooley, ed., 1992, New York NY, American Institute of Physics,

[8] Namur-Empfehlung, NE107, "Selbstüberwachung und Diagnose von Feldgeräten“"

[9] M.Henry: Sensor validation - principles and standards, atp international, 3 (2005) No.2, München, Oldenbourg Verlag 